\title{
Interpolating Sparsely Corrupted Signals in Micrometeorology
}

\author{
Carlos Ramirez ${ }^{1}$, Miguel Argáez ${ }^{1}$, Aline Jaimes ${ }^{2}$ \& Craig E. Tweedie ${ }^{2}$ \\ ${ }^{1}$ Computational Science Program, The University of Texas at El Paso, El Paso, Texas, United States \\ ${ }^{2}$ Environmental Science and Engineering Program, The University of Texas at El Paso, El Paso, Texas, United \\ States \\ Correspondence: Carlos Ramirez, Computational Science Program, The University of Texas at El Paso, El Paso, \\ TX., United States. E-mail: caramirezvillamarin@ miners.utep.edu
}

\author{
Received: November 11, 2013 Accepted: March 15, 2014 Online Published: April 9, 2014 \\ doi:10.5539/jmr.v6n2p10 URL: http://dx.doi.org/10.5539/jmr.v6n2p10
}

The authors thank the financial support from the U.S. Army Research Laboratory Grant No. W911NF-07-2-0027. This research is partially supported by the NSF Grant No. HRD-073482 and the National Council of Science and Technology - CONACyT

\begin{abstract}
In real applications where data acquisition is carried out under extreme conditions, post-processing techniques for systematic corrections are of critical importance. In micrometeorological studies, it is often the case that acquired data contains both missing information and impulse noise due to instrumentation failure, data transmission and data rejection for quality assurance. In this work, we propose a simple algorithm based on an $\ell_{1}-\ell_{1}$ variational formulation that simultaneously suppresses impulse noise and interpolates missing information. Our approach consists of relaxing the objective function in the variational formulation with a strictly convex and continuously differentiable function that depends on a regularization parameter. We solve a sequence of strictly convex optimization subproblems as the regularization parameter goes to zero, converging to the solution of the original problem. Numerical experiments on real micrometeorological datasets are conducted showing the effectiveness of the proposed approach. Furthermore, a convergence analysis is presented providing theoretical guaranties of our method.
\end{abstract}

Keywords: impulse noise, denoising, interpolation, $\ell_{1}-\ell_{1}$ minimization

\section{Introduction}

In Micrometeorology, the tower-based Eddy Covariance (EC) method is widely used to measure the exchange of carbon dioxide, water vapor, and energy between terrestrial ecosystems and the atmosphere. From EC measurements, patterns and controls of ecosystem exchange are typically deduced and the potential for environmental impacts such as desertification of arid rangelands, deforestation of tropical forest, and warming of tundra landscapes are assessed.

Importantly, the EC method can be limited by environmental variability (hilly areas, heterogeneous canopy height, downwind turbulence), extreme environmental events (freezes, snow, dust storms, hail, lightening) and general instrumentation requirements (insufficient or none power supply, incorrect calibration, transmission), thereby producing incomplete and sparsely corrupted (spiky) datasets (Moncrieff et al., 1996).

In order to compute accurate representations of land-atmosphere trace gas fluxes and energy exchange so that reliable conclusions can be drawn, raw EC data needs to be corrected and processed. As a consequence, gap filling and de-spiking procedures need to be established (Falge et al., 2001).

Several gap filling methods have been developed and tested by the micrometeorological community. These methods include non-linear regression, Kalman filtering, artificial neural networks, mean diurnal variation, and the recently proposed wavelet sparse representation (Ramirez et al., 2012). A comprehensive comparison of such methods can be found in (Moffat et al., 2007). These methods, however, only address the interpolation process, and consider the removal of spikes as a separate task. In this work, we propose a simple algorithm based on an 
an $\ell_{1}-\ell_{1}$ variational formulation that simultaneously suppresses the impulse noise (i.e., sparse corruption) and interpolates the missing information.

Although the $\ell_{1}-\ell_{1}$ variational model has been a subject of interest for the signal processing community in recent years, most of this interest has been focused on image processing applications such as denoising and deblurring in the presence of impulse noise (Bar \& Kiryati, 2006; Nikolova, 2004). To the best of our knowledge, research aimed at interpolating signals in the presence of impulse noise has been very limited, perhaps with the exception of Wang et al. (2011) and Yan (2011) which, nonetheless, fall in the image processing setting.

This paper is organized as follows. In Section 2, we present the mathematical formulation of the problem. In Section 3, we describe our algorithm and give theoretical guaranties that motivate the numerical implementation. In Section 4, we present numerical results involving real micrometeorological data supporting the proposed method. In Section 5, we present the relevance of this study and how it relates to previous work. Finally, Section 6 presents some concluding remarks.

\section{Problem Formulation}

An observed signal $b \in \mathbb{R}^{m}$ is obtained after the original data set $u \in \mathbb{R}^{n}$ is subject to a data loss process followed by a selective corruption in some elements of $b$. This degradation process can be modeled as an underdetermined linear system

$$
b=H u+v,
$$

where $H \in \mathbb{R}^{m \times n}(m<n)$ is constructed by removing from the $n \times n$ identity matrix, the $n-m$ rows associated with the missing data. The vector $v \in \mathbb{R}^{m}$ is additive impulse or sparse noise in which the nonzero elements are drawn from a normal distribution with zero mean and standard deviation $\sigma_{0}$. Thus, the difference $H u-b$ must be sparse at the solution, so that the fidelity term $\|H u-b\|_{1}$ becomes a suitable choice for measuring the discrepancy between $b$ and $H u$. Furthermore, if $u$ is assumed to be sparse in a given basis $\Psi$, that is $u=\Psi x$ with $x$ sparse, this prior knowledge can be imposed at the solution with an $\ell_{1}$ penalty term. Therefore, in order to recover or estimate the original signal (data), we formulate the following unconstrained optimization problem

$$
\min _{x} f(x)=\|A x-b\|_{1}+\lambda\|x\|_{1}
$$

where $A=H \Psi$ and $\lambda \in\left(0,\left\|A^{T} \operatorname{sig}(b)\right\|_{\infty}\right)$ is a penalization parameter that balances the fidelity of the solution in the $\ell_{1}$ norm sense, while promoting sparsity in $x$. The operator $\operatorname{sig}(\cdot)$ is applied component wise, and returns the sign of the argument.

\section{Methodology}

The major challenge of solving problem (1) is dealing with the non differentiability of the $\ell_{1}$ norm. While several numerical methods have proliferated for solving (1) in which the fidelity term is measured with the $\ell_{2}$ norm, e.g. (Chen et al., 2001; Kim et al., 2007; Figueiredo et al., 2007; Wright et al., 2008; Argaez et al., 2011; Becker et al., 2010), research efforts aimed at solving (1) as it is presented here have been more limited. Such works include Fu et al. (2006), where problem (1) is posed as a linear programming problem and solved using interior point methods; Nikolova (2004), who considers the denoising case, i.e. $A=I$, and proposes a regularization term based upon local regularity replacing the second term in (1) by

$$
\frac{1}{2} \sum_{i=1}^{n} \sum_{j \in \mathcal{N}_{i}}\left|x_{i}-x_{j}\right|
$$

where $\mathcal{N}_{i}$ is a neighborhood of $x_{i}$. Finally, Bar and Kiryati (2006) consider the image deconvolution problem in the presence of impulse noise using an $\ell_{1}$ fidelity term, and a Mumford-Shah functional to promote piece-wise regularity as well as edge preserving features.

In order to solve (1), we extend our ideas reported in (Ramirez \& Argaez, 2013) where the non differentiability of the $\ell_{1}$ norm is overcome by approximating the absolute value with a continuously differentiable and strictly convex function that depends on a regularization parameter $\mu_{k}$. More specifically, $|x| \approx \sqrt{x^{2}+\mu_{k}}$ where $\mu_{k} \rightarrow 0$ as $k \rightarrow \infty$. Therefore, our strategy consists of solving a sequence of strictly convex optimization subproblems of the form

$$
\min _{x} f_{\mu_{k}}(x)=\sum_{i=1}^{m} \sqrt{\left(a_{i}^{T} x-b_{i}\right)^{2}+\mu_{k}}+\lambda \sum_{i=1}^{n} \sqrt{x_{i}^{2}+\mu_{k}}
$$


as $k \rightarrow \infty$.

The main difference of this formulation and the one presented in (Ramirez \& Argaez, 2013) is that the latter assumes normality in the distribution of residuals $r_{i}=a_{i}^{T} x-b_{i}$ whereas in this work the residual vector $r$ is modeled as sparse vector due to the presence of spikes in the measurements $b$.

Now, notice that for any $\mu_{k}>0$, Problem (2) is strictly convex since the Hessian matrix

$$
\nabla^{2} f_{\mu_{k}}(x)=\mu_{k} A^{T} D_{r}^{-\frac{3}{2}} A+\operatorname{diag}\left(\frac{\lambda \mu_{k}}{\left(x_{i}^{2}+\mu_{k}\right)^{\frac{3}{2}}}\right)
$$

is positive definite. Here $D_{r}=\operatorname{diag}\left(r_{i}^{2}+\mu_{k}\right)$ and $r_{i}=a_{i}^{T} x-b_{i}$. In fact, for any non-zero $d \in \mathbb{R}^{n}$, we have that

$$
\begin{aligned}
d^{T} \nabla^{2} f_{\mu_{k}}(x) d & =\mu_{k} d^{T} A^{T} D_{r}^{-\frac{3}{2}} A d+\lambda \mu_{k} \sum_{i=1}^{n} \frac{d_{i}^{2}}{\left(x_{i}^{2}+\mu_{k}\right)^{\frac{3}{2}}} \\
& =\mu_{k}\left\|D_{r}^{-\frac{3}{4}} A d\right\|_{2}^{2}+\lambda \mu_{k} \sum_{i=1}^{n} \frac{d_{i}^{2}}{\left(x_{i}^{2}+\mu_{k}\right)^{\frac{3}{2}}} \\
& \geq \lambda \mu_{k} \sum_{i=1}^{n} \frac{d_{i}^{2}}{\left(x_{i}^{2}+\mu_{k}\right)^{\frac{3}{2}}} \\
& >0 .
\end{aligned}
$$

Additionally, $f_{\mu_{k}}$ is proper since $f_{\mu_{k}}(x)<\infty$ for at least one $x \in \mathbb{R}^{n}$ (e.g. $x=0$ ), and $f_{\mu_{k}}(x)>-\infty$ for all $x \in \mathbb{R}^{n}$ (in fact, $f_{\mu_{k}}$ is bounded below by 0). Finally, $f_{\mu_{k}}$ is coercive since $\lim _{\|x\| \rightarrow \infty} f_{\mu_{k}}(x)=+\infty$. Therefore for $\mu_{k}>0$, the optimal set of (2) is nonempty and has at most one element. Put differently, the global minimizer $x_{\mu_{k}}$ of (2) exists and is unique.

\subsection{Optimality Conditions}

The partial derivative of $f_{\mu_{k}}(x)$ with respect to $x_{i}$ is

$$
\frac{\partial f_{\mu_{k}}(x)}{\partial x_{i}}=\sum_{j=1}^{m} \frac{a_{j i}\left(a_{j}^{T} x-b_{j}\right)}{\sqrt{\left(a_{j}^{T} x-b_{j}\right)^{2}+\mu_{k}}}+\lambda \frac{x_{i}}{\sqrt{x_{i}^{2}+\mu_{k}}} .
$$

Using matrix notation, the gradient of $f_{\mu_{k}}(x)$ is written more compactly as

$$
\nabla f_{\mu_{k}}(x)=A^{T} D_{r}^{-\frac{1}{2}}(A x-b)+\lambda D_{x}^{-\frac{1}{2}} x,
$$

where $D_{r}=\operatorname{diag}\left(r_{i}^{2}+\mu_{k}\right)$ with $r_{i}=a_{i}^{T} x-b_{i}$, and $D_{x}=\operatorname{diag}\left(x_{i}^{2}+\mu_{k}\right)$.

Consequently, the unique minimizer of (2) is characterized by the following system of nonlinear equations

$$
A^{T} D_{r}^{-\frac{1}{2}} A x-A^{T} D_{r}^{-\frac{1}{2}} b+\lambda D_{x}^{-\frac{1}{2}} x=0 .
$$

Noticing that $\lambda D_{x}^{-\frac{1}{2}}$ is a positive definite matrix, we can write (3) as a fixed point equation of the form

$$
x=\mathcal{F}(x),
$$

where $\mathcal{F}(x)=\left(A^{T} D_{r}^{-\frac{1}{2}} A+\lambda D_{x}^{-\frac{1}{2}}\right)^{-1} A^{T} D_{r}^{-\frac{1}{2}} b$. Therefore, given an initial point $x_{-}$, we solve for $x$ the following linear system

$$
\left(A^{T} D_{r_{-}}^{-\frac{1}{2}} A+\lambda D_{x_{-}}^{-\frac{1}{2}}\right) x=A^{T} D_{r_{-}}^{-\frac{1}{2}} b,
$$

where $D_{r_{-}}=\operatorname{diag}\left(\left(r_{-}\right)_{i}^{2}+\mu_{k}\right)$ with $\left(r_{-}\right)_{i}=a_{i}^{T} x_{-}-b_{i}$, and $D_{x_{-}}=\operatorname{diag}\left(\left(x_{-}\right)_{i}^{2}+\mu_{k}\right)$. We continue this process iteratively until two consecutive points $x_{-}$and $x$ are sufficiently close. This fixed point scheme motivates the algorithmic framework presented below.

\subsection{The Algorithm}

We propose a modification of the Fixed Point Least Squares-Preconditioned Conjugate Gradient (FPLS_PCG) algorithm presented in (Ramirez \& Argaez, 2013) for solving Problem (1). 


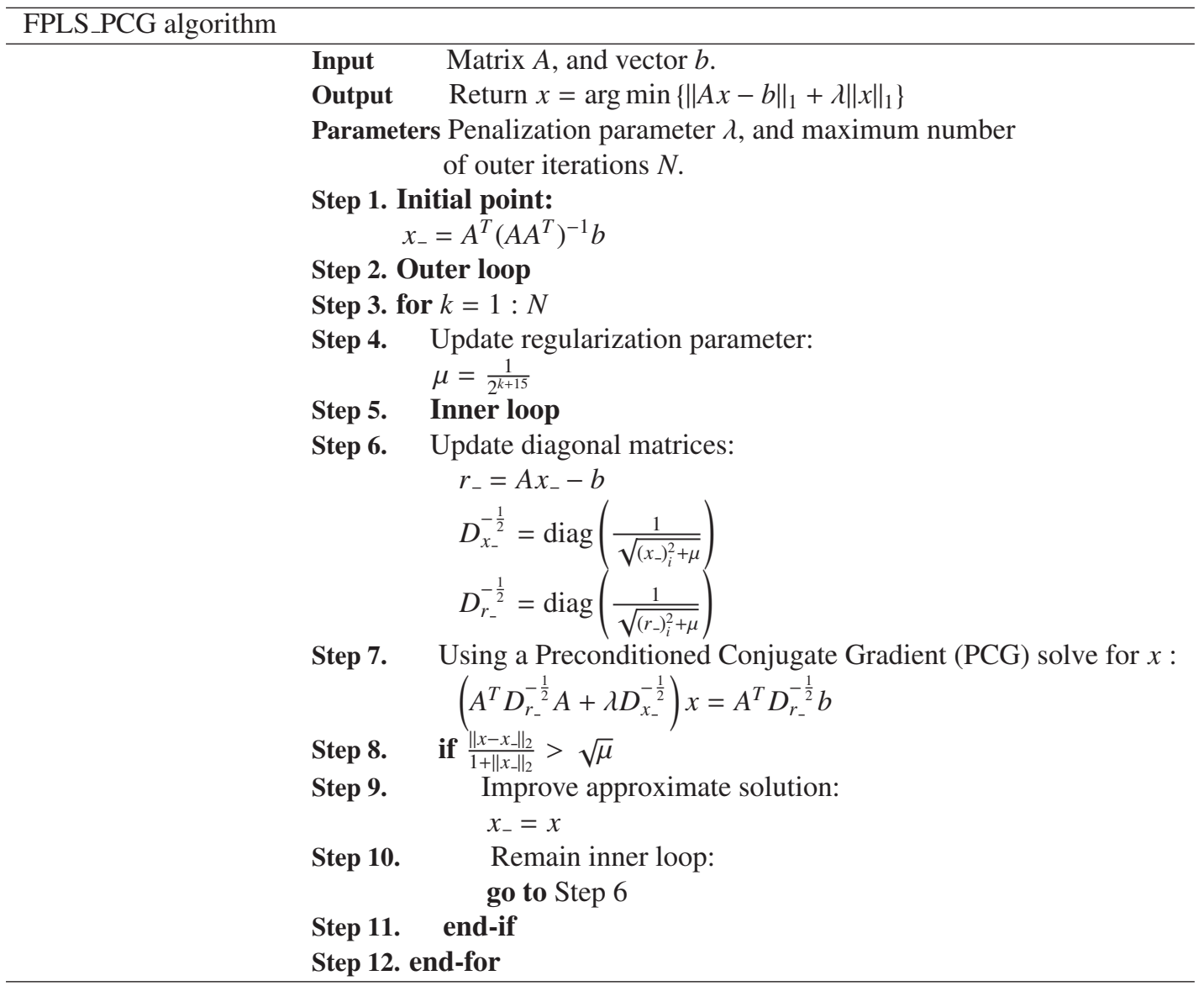

Before concluding this section, we want to comment on the following two aspects. Firstly, it is important to realize that the main difference between the FPLS_PCG algorithm presented here and the one in (Ramirez \& Argaez, 2013) is in the Steps 6 and 7. In these Steps there is an extra re-weighting matrix $D_{r}$ associated with the residual vector $r=A x-b$ which accounts for the non-Gaussian noise present in the measurement vector $b$. Secondly, although any monotonically decreasing sequence $\left\{\mu_{k}\right\}_{k}$ is theoretically a guarantee for convergence to the optimal set of (1) as proved in next section, numerical experiments have demonstrated that the choice $\mu_{k}=\frac{1}{2^{k+15}}$ performs well in practice for a broad set of problems. This is, however, not a general rule and the choice of $\left\{\mu_{k}\right\}_{k}$ can be considered a non-sensitive parameter.

\subsection{Convergence Analysis}

We show that any limit point of the sequence of minimizers $\left\{x_{\mu_{k}}\right\}_{\mu_{k} \rightarrow 0}$ of Problem (2) is contained in the optimal set of Problem (1).

Notice that the family of global minimizers $\left\{x_{\mu}\right\}$ as $\mu \rightarrow 0$ is bounded. This is a consequence of the bound

$$
\begin{aligned}
\lambda \sum_{i=1}^{n}\left|\left(x_{\mu}\right)_{i}\right| & \leq \lambda \sum_{i=1}^{n} \sqrt{\left(x_{\mu}\right)_{i}^{2}+\mu} \\
& \leq \sum_{i=1}^{m} \sqrt{\left(a_{i}^{T} x_{\mu}-b_{i}\right)^{2}+\mu}+\lambda \sum_{i=1}^{n} \sqrt{\left(x_{\mu}\right)_{i}^{2}+\mu} \\
& \leq m \sqrt{\|b\|_{\infty}^{2}+\mu}+n \lambda \sqrt{\mu},
\end{aligned}
$$

where the last inequality follows because $f_{\mu}\left(x_{\mu}\right) \leq f_{\mu}(x)$ for all $x \in \mathbb{R}^{n}$, in particular for $x=0$. Therefore,

$$
\left\|x_{\mu}\right\|_{1} \leq \frac{m}{\lambda} \sqrt{\|b\|_{\infty}^{2}+\mu}+n \sqrt{\mu}
$$

and the family $\left\{x_{\mu}\right\}_{\mu \rightarrow 0}$ is bounded. Hence, there exists a convergent subsequence of $\left\{x_{\mu_{k}}\right\}_{k}$ which for simplicity 
will be denoted by $\left\{x_{\mu_{k}}\right\}_{k}$, and whose limit is written as

$$
\bar{x}=\lim _{k \rightarrow \infty} x_{\mu_{k}} .
$$

Now, since $x_{\mu_{k}}$ is the global minimizer of $f_{\mu_{k}}(x)$, then we have

$$
\sum_{i=1}^{m} \sqrt{\left(a_{i}^{T} x_{\mu_{k}}-b_{i}\right)^{2}+\mu}+\lambda \sum_{i=1}^{n} \sqrt{\left(x_{\mu_{k}}\right)_{i}^{2}+\mu_{k}} \leq \sum_{i=1}^{m} \sqrt{\left(a_{i}^{T} x^{*}-b_{i}\right)^{2}+\mu}+\lambda \sum_{i=1}^{n} \sqrt{\left(x^{*}\right)_{i}^{2}+\mu_{k}},
$$

where $x^{*}=\arg \min f(x)$ is a solution of (1).

Taking the limit as $\mu_{k} \rightarrow 0$ in the above expression and by continuity it yields

$$
\|A \bar{x}-b\|_{1}+\lambda\|\bar{x}\|_{1} \leq\left\|A x^{*}-b\right\|_{1}+\lambda\left\|x^{*}\right\|_{1} .
$$

Consequently, $f(\bar{x}) \leq f\left(x^{*}\right)$ and therefore $\bar{x}$ is a minimizer of $f(x)$. In other words, any limit point of the sequence of minimizers $\left\{x_{\mu_{k}}\right\}_{\mu_{k} \rightarrow 0}$ of Problem (2), is contained in the optimal set of (1).

\section{Numerical Results}

In this section we use datasets from the Eddy Covariance tower established by UTEP's Systems Ecology Lab (SEL) and Cyber-ShARE center at the Jornada Basin Experimental Range, Las Cruces, New Mexico (USA). We select a 31-month data set of four 30-min averaged micrometeorological variables (Carbon Dioxide- $\mathrm{CO}_{2}, \mathrm{Water}$ Vapor- $\mathrm{H}_{2} \mathrm{O}$, Air Temperature-AT and Atmospheric Pressure-PRESS). The variables $\mathrm{H}_{2} \mathrm{O}$ and AT have 44.953 measurements where a total of 5.303 are missing or rejected, and nearly $1 \%$ are corrupted by impulse noise. On the other hand, the variables $\mathrm{CO}_{2}$ and PRESS whose sensor platform was deployed at a later date, have 42.363 measurements where a total of 4.229 are missing or rejected, with the same percentage of impulse noise.
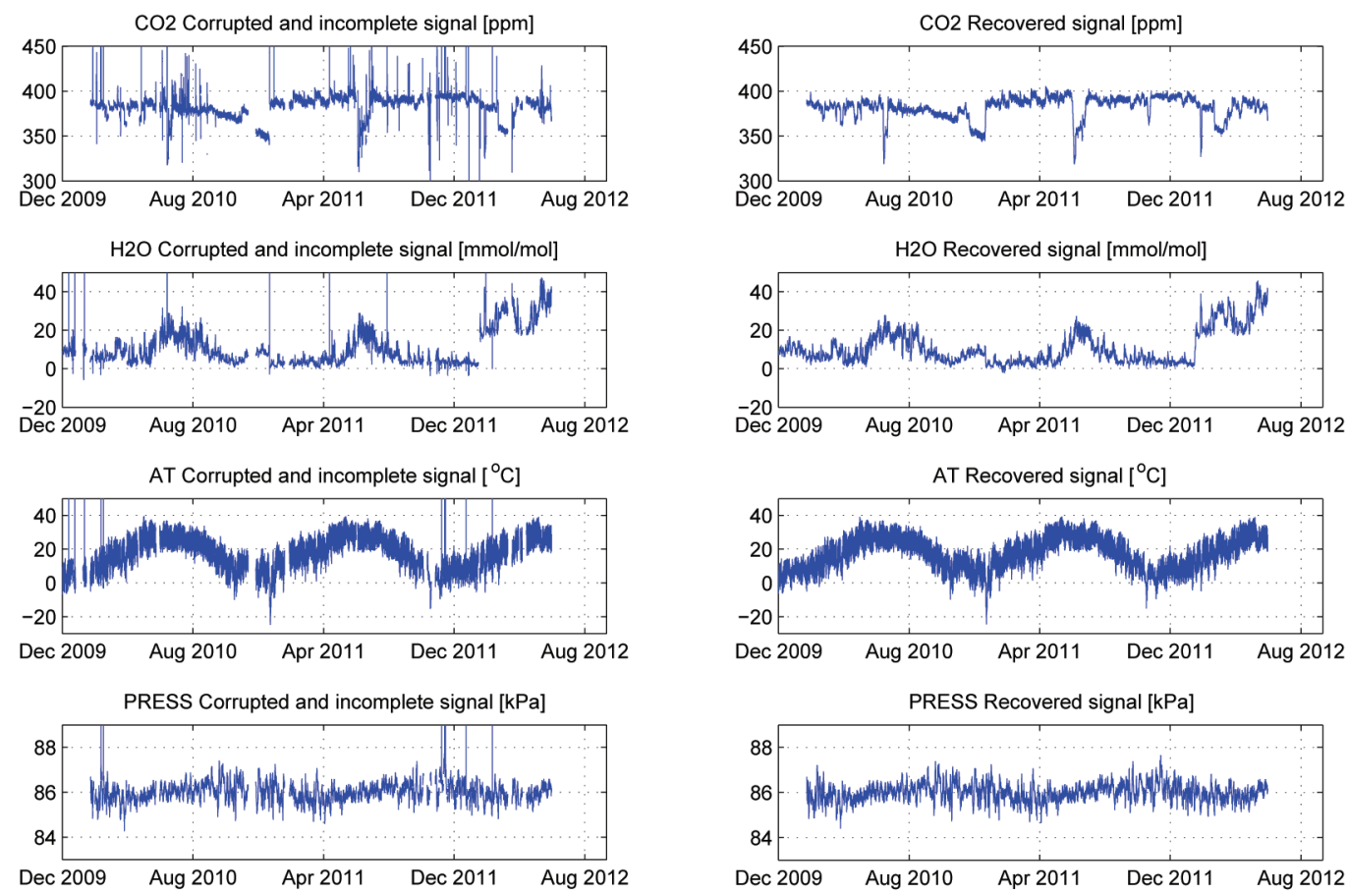

Figure 1. (Left) Corrupted and incomplete datasets of $\mathrm{CO}_{2}, \mathrm{H}_{2} \mathrm{O}$, AT and PRESS respectively. (Right) Recovered datasets after applying the FPLS_PCG algorithm

Our aim is to simultaneously estimate the missing information while suppressing the impulse or sparse noise. We further assume that the variable of interest can be sparsified by the Discrete Cosine Transform (DCT). This is a reasonable assumption in micrometeorology, since the variable of interest derives most of its features from 
seasonal and diurnal changes. Therefore, we solve Problem (1) where $\Psi$ is the DCT transform, $H$ is the subsampled identity matrix associated with the missing information, and $b$ is the observed data. Figure 1 (left) shows the incomplete and corrupted datasets corresponding to $\mathrm{CO}_{2}, \mathrm{H}_{2} \mathrm{O}$, AT and PRESS respectively. Figure 1 (right) shows a successfully recovered dataset after running our FPLS_PCG algorithm for all four variables.

In order to assess the error associated with the increase of missing and corrupted elements, we take a 512-length fraction of uncorrupted data denoted by $u^{*}$, and solve the recovery problem after artificial gaps and impulse noise are randomly added. The added artificial gaps range from 5\% up to 20\%, and the impulse noise from $1 \%$ up to $3 \%$. In each case, we compute the 2 -norm error defined by

$$
\text { 2-norm error }=\frac{\left\|\hat{u}-u^{*}\right\|_{2}}{\left\|u^{*}\right\|_{2}},
$$

where $\hat{u}$ is the recovered signal. This experiment set up is run one hundred times, and the averaged results are reported in Table 1, which shows that even with $20 \%$ of missing pixels and $3 \%$ of impulse noise, a successful recovery is achieved. Figure 2 illustrates the recovery for the AT data set where $10 \%$ of the data is missing, and $2 \%$ of the elements are affected by impulse noise.

Table 1. Scalability 2-norm error for different percentages of artificial gaps and impulse noise in a 512-length signal

\begin{tabular}{|c|c|c|c|c|c|c|}
\hline & \multicolumn{3}{|c|}{$5 \%$} & \multicolumn{3}{|c|}{$10 \%$} \\
\hline & $1 \%$ & $2 \%$ & $3 \%$ & $1 \%$ & $2 \%$ & $3 \%$ \\
\hline $\mathrm{CO}_{2}$ & $5.01 \mathrm{E}-4$ & $1.02 \mathrm{E}-3$ & $1.10 \mathrm{E}-3$ & $6.68 \mathrm{E}-4$ & $7.84 \mathrm{E}-4$ & 7.14E-4 \\
\hline $\mathrm{H}_{2} \mathrm{O}$ & $1.21 \mathrm{E}-2$ & $1.29 \mathrm{E}-2$ & $2.04 \mathrm{E}-2$ & $1.53 \mathrm{E}-2$ & $3.37 \mathrm{E}-2$ & $4.65 \mathrm{E}-2$ \\
\hline PRESS & $2.64 \mathrm{E}-5$ & $2.40 \mathrm{E}-5$ & $2.37 \mathrm{E}-5$ & $4.25 \mathrm{E}-5$ & $4.16 \mathrm{E}-5$ & $5.43 \mathrm{E}-5$ \\
\hline \multirow[t]{3}{*}{$\mathrm{AT}$} & $5.90 \mathrm{E}-2$ & $5.91 \mathrm{E}-2$ & $5.90 \mathrm{E}-2$ & $7.22 \mathrm{E}-2$ & $7.55 \mathrm{E}-2$ & $9.15 \mathrm{E}-2$ \\
\hline & \multicolumn{3}{|c|}{$15 \%$} & \multicolumn{3}{|c|}{$20 \%$} \\
\hline & $1 \%$ & $2 \%$ & $3 \%$ & $1 \%$ & $2 \%$ & $3 \%$ \\
\hline $\mathrm{CO}_{2}$ & $9.13 \mathrm{E}-4$ & $8.63 \mathrm{E}-4$ & $7.95 \mathrm{E}-4$ & $9.53 \mathrm{E}-4$ & $1.09 \mathrm{E}-3$ & $8.91 \mathrm{E}-4$ \\
\hline $\mathrm{H}_{2} \mathrm{O}$ & $1.88 \mathrm{E}-2$ & $2.39 \mathrm{E}-2$ & $4.67 \mathrm{E}-2$ & $2.41 \mathrm{E}-2$ & $4.14 \mathrm{E}-2$ & $6.89 \mathrm{E}-2$ \\
\hline PRESS & $3.76 \mathrm{E}-5$ & 5.37E-5 & 4.45E-5 & $6.21 \mathrm{E}-5$ & $5.56 \mathrm{E}-5$ & $5.24 \mathrm{E}-5$ \\
\hline AT & $1.13 \mathrm{E}-1$ & $1.15 \mathrm{E}-1$ & $1.24 \mathrm{E}-1$ & $1.47 \mathrm{E}-1$ & $2.86 \mathrm{E}-1$ & $3.34 \mathrm{E}-1$ \\
\hline
\end{tabular}
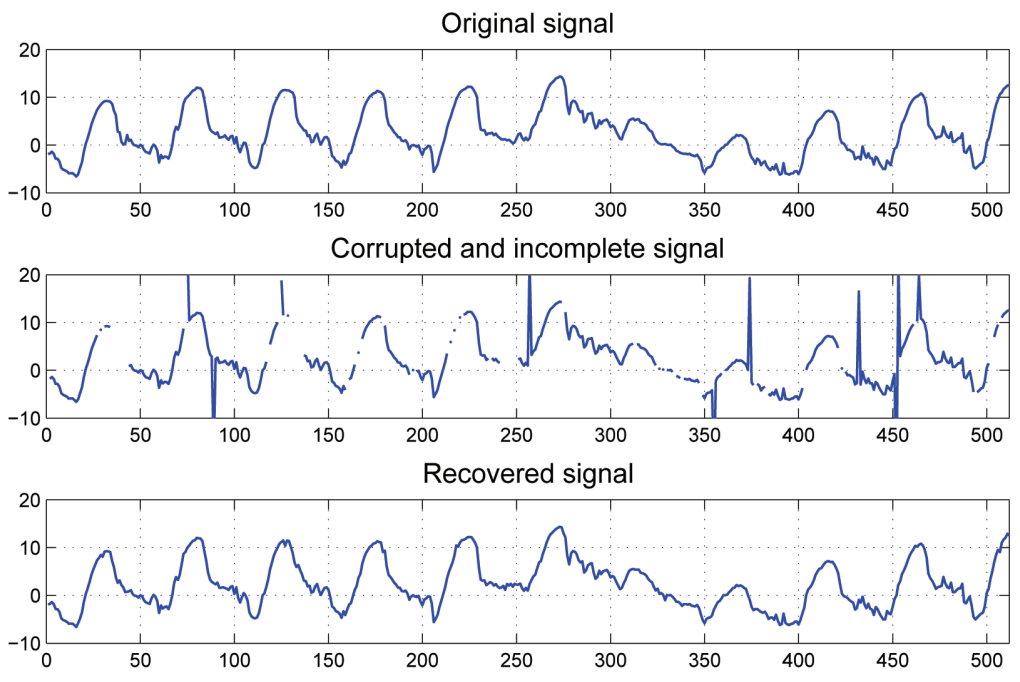

Figure 2. (Top) Original Signal (AT). (Middle) Incomplete and corrupted signal. 10\% of the data has been removed, and a $2 \%$ of impulse noise has been added. (Bottom) Recovered signal after applying the FPLS_PCG algorithm with a relative 2-norm error of 0.075 


\section{Discussion}

The work presented here focuses on recovering degraded digital signals based on a sparse-inducing variational formulation. While numerous algorithms and methodologies have been proposed to solve this problem in the presence of Gaussian noise e.g. (Chen et al, 2001; Kim et al., 2007; Figueiredo et al., 2007; Wright et al., 2008; Argaez et al., 2011; Becker et al., 2010), strategies addressing the case of impulse or sparse noise are more limited. Recently, the works of Nikolova (2004), Fu et al. (2006) and Bar et al. (2006) studied the recovery problem in the presence of impulse noise but in an image processing setting and for complete datasets. Our work, on the other hand, is aimed at solving the recovery problem in the presence of impulse noise where the set of observations is not complete. Prior and related works in this direction include Wang et al. (2011) and Yan (2011), but consider Gaussian noise and fall in the image processing setting. Furthermore, our work is an extension of Ramirez et al. (2012) where only the interpolation process is considered. Last but not least, this work constitutes an effort to bridge state-of-the-art signal processing techniques with other scientific disciplines, in this case, micrometeorology.

\section{Concluding Remarks}

In this paper, we propose a simple algorithm for recovering incomplete micrometeorological signals that are further corrupted by impulse or sparse noise. The underlying approach consists of solving an $\ell_{1}-\ell_{1}$ variational problem in which the absolute value is relaxed with a continuously differentiable and strictly convex function that depends on a regularization parameter. We solve a sequence of strictly convex optimization subproblems as the regularization parameter goes to zero. A convergence analysis is presented showing the theoretical guaranties of the proposed method. Furthermore, numerical experiments on real micrometeorological datasets are conducted demonstrating the effectiveness of the proposed algorithm.

\section{References}

Argaez, M., Ramirez, C., \& Sanchez, R. (2011). An $\ell_{1}$ algorithm for underdetermined systems and applications. Annual Meeting of the North American Fuzzy Information Processing Society (NAFIPS 2011). http://dx.doi.org/10.1109/NAFIPS.2011.5752016

Bar, L., \& Kiryati, N. (2006). Image Deblurring in Presence of Impulsive Noise. Int. J. Comput. Vision, 70(3), 279-298.

Becker, S., Bobin, J., \& Candès, E. (2010). NESTA: a fast and accurate first-order method for sparse recovery. SIAM J. on Imaging Sciences, 4(1), 1-39.

Chen, S., Donoho, D., \& Saunders, M. (2001). Atomic Decomposition by Basis Pursuit. SIAM Review, 43(1), 129-159.

Falge, E., Baldocchi, D., Olson, R., Anthoni, P., Aubinet, M., Bernhofer, C., ... Wofsy, S. (2001). Gap filling strategies for defensible annual sums of net ecosystem exchange. Agricultural and Forest Meteorology, 107(1), 43-69.

Figueiredo, M., Nowak, R., \& Wright, S. (2007). Gradient projection for sparse reconstruction: Application to compressed sensing and other inverse problems. IEEE J. Sel. Topics Signal Process, 1(4), 586-597.

Fu, H., Ng, M., Nikolova, M., \& Barlow, J. (2006). Efficient Minimization Methods of Mixed $\ell_{2}-\ell_{1}$ and $\ell_{1}-\ell_{1}$ Norms for Image Restoration. SIAM Journal in Scientific Computing, 27(6), 1881-1902.

Kim, S., Koh, K., Lustig, M., Boyd, S., \& Gorinvesky, D. (2007). An interior-point method for large-scale $\ell_{1-}$ regularized least squares. IEEE J. Sel. Topics Signal Process, 1(4), 606-617.

Moffat, A. M., Papale, D., Reichstein, M., Hollinger, D., Richardson, A., Barr, A., ... Stauch, V. (2007). Comprehensive comparison of gap-filling techniques for eddy covariance net carbon fluxes. Agricultural and Forest Meteorology, 147(3-4), 209-232.

Moncrieff, J., Malhi, Y., \& Leuning, R. (1996). The propagation of errors in long-term measurements of landatmosphere fluxes of carbon and water. Global Change Biology, 2(3), 231-240.

Nikolova, M. (2004). A Variational Approach to Remove Outliers and Impulse Noise. Journal of Mathematical Imaging and Vision, 20, 99-120.

Ramirez, C., \& Argaez, M. (2013). An $\ell_{1}$ minimization algorithm for non-smooth regularization in image processing. Signal, Image and Video Processing. http://dx.doi.org/10.1007s11760-013-0454-1 
Ramirez, C., Argaez, M., Jaimes, A., \& Tweedie, C. E. (2012). Image inpainting in micrometeorological analysis. IEEE proceedings of the IEEE International Conference in Image Processing (ICIP 2012) (pp. 1725-1728).

Wang, Y., Szlam, A., \& Lerman G. (2011). Robust Locally Linear Analysis with Applications to Image Denoising and Blind Inpainting. Technical Report 2379 Institute for Mathematics and its Applications, University of Minnesota.

Wright, S., Nowak, R., \& Figueiredo, M. (2008). Sparse reconstruction by separable approximation. IEEE International Conference on Acoustics, Speech and Signal Processing, ICASSP 2008. (pp. 3373-3376). Las Vegas, NV.

Yang, M. (2011). Restoration of Images Corrupted by Impulse Noise using Blind Inpainting and $\ell_{0}$ Norm. Technical Report UCLA CAM report 11-72.

\section{Copyrights}

Copyright for this article is retained by the author(s), with first publication rights granted to the journal.

This is an open-access article distributed under the terms and conditions of the Creative Commons Attribution license (http://creativecommons.org/licenses/by/3.0/). 